\title{
Efecto del bypass gástrico en la esofagitis erosiva en pacientes con obesidad mórbida
}

\author{
Attila Csendes J, FACS, Ana María Burgos L, Gladys Smok S, \\ Patricio Burdiles P.
}

\section{Effects of gastric bypass on erosive esophagitis in obese subjects}

Background: Obesity is an important risk for pathological gastroesophageal reflux. Aim: To assess the effects of gastric bypass on obese subjects with erosive esophagitis. Patients and methods: Sixty two morbid obese subjects (aged 16 to 70 years, 41 females) with erosive esophagitis at the moment of surgery were studied. These patients were subjected to a gastric bypass with gastric resection. They were followed with upper gastrointestinal endoscopy every one year, looking for the presence of erosions or ulcers in the distal esophagus. Results: The mean follow up period was 21 months. Prior to surgery all patients had heartburn or regurgitation and at two years after surgery, 97\% were asymptomatic. Esophagitis was found in 97 and $6.5 \%$ before and after surgery, respectively. Two patients had an esophageal ulcer, that healed on follow up endoscopy. There was a $72 \%$ reduction of overweight on late follow up. Conclusions: Gastric bypass is effective to control pathological gastroesophageal reflux in patients with morbid obesity (Rev Méd Chile 2006; 134: 285-90).

(Key words: Bariatric surgery; Gastric bypass; Obesity, morbid)

Recibido el 18 de mayo, 2005. Aceptado el 28 de agosto, 2005.

Departamento de Cirugía, Hospital Clínico Universidad de Chile.

$\mathrm{L}$ a obesidad es un importante factor en la génesis del reflujo gastroesofágico crónico patológico $^{1-3}$. Esto trae como consecuencias trastornos anatómicos e histológicos del esófago,

Correspondencia a: Dr. Attila Csendes, FACS (Hon). Departamento de Cirugía, Hospital Clínico J.J. Aguirre. Santos Dumont 999, Santiago, Chile. Fono: 56-2-7774387. Fax: 562-7775043. E mail: acsendes@med.uchile.cl como esofagitis erosiva, esófago de Barrett y hernia hiatal ${ }^{4}$. En pacientes con obesidad mórbida, el bypass gástrico es el procedimiento gold standard para el tratamiento quirúrgico de esta afección $^{5-9}$. Esta operación es una técnica antirreflujo muy completa, con una significativa mejoría subjetiva y clínica de los pacientes ${ }^{10-17}$.

El objetivo del presente estudio prospectivo fue determinar el efecto del bypass gástrico sobre la presencia de esofagitis erosiva en un grupo de pacientes con obesidad mórbida. 


\section{Material y MÉtodo}

Pacientes. El presente estudio prospectivo comenzó el 9 de agosto de 1999 y se cerró el 10 de enero de 2005 , incluyendo a 570 pacientes con obesidad mórbida sometidos a cirugía bariátrica. De ellos, en 451 pacientes se realizó endoscopia y toma de biopsias preoperatorias, ya que en los 119 pacientes restantes sólo se realizó endoscopia. De estos 451 pacientes, se comprobó la presencia de erosiones esofágicas en 121 pacientes (26,8\%). De ellos, en 69 pacientes hay control endoscópico de más de 18 meses, que constituyen el presente estudio, ya que 50 tienen un seguimiento más corto. Se excluyeron pacientes con esófago de Barrett y metaplasia intestinal cardial (total 17 casos).

El grupo analizado comprende 45 mujeres (65\%) y 24 hombres (35\%), con una edad promedio de 37,5 años \pm 12 (rango 16 a 70). Todos los pacientes tenían una obesidad mórbida, es decir, más de 40 $\mathrm{kg} / \mathrm{m}^{2}$ o más de $35 \mathrm{~kg} / \mathrm{m}^{2}$ con alguna comorbilidad importante, como diabetes mellitus tipo II, hipertensión arterial o dislipidemia. Hubo 15 pacientes (22\%) con un IMC menor a $40 \mathrm{~kg} / \mathrm{m}^{2}, 46$ pacientes (67\%) con un IMC entre 40 y $50 \mathrm{~kg} / \mathrm{m}^{2}$ y 8 pacientes (11\%) con un IMC sobre $50 \mathrm{~kg} / \mathrm{m}^{2}$. Ningún paciente estaba recibiendo fármacos inhibidores de la secreción ácida antes ni después de la cirugía.

Procedimientos endoscópicos. Todos los pacientes fueron sometidos a un estudio endoscópico, 10 a 14 años previos a la cirugía y a 1 ó 2 estudios después de la operación (a los 12 y 24 meses). El instrumento empleado es un endoscopio GIFXQ20 (Olympus, Tokio). Hubo un especial cuidado en evitar el efecto de "empuje" o "tracción" del instrumento. Se evaluó la presencia de erosiones del esófago distal, antes y después de la cirugía. Se evaluó también la presencia de úlcera esofágica, que es un exudado con bordes nítidos habitualmente situado en la unión de mucosas escamoso columnar.

Estudio histológico. En todos los pacientes se tomaron 2 a 3 muestras $5 \mathrm{~mm}$ distal del cambio de mucosas escamoso columnar, que se llamaron «biopsias yuxtacardiales". Estas muestras se sumergieron de inmediato en formalina al 10\% y se enviaron a tinción con hematoxilina eosina, y azul de Alcian a pH 2,5. Como se comentó previamente, pacientes con metaplasia intestinal del esófago distal o cardias se excluyeron del presente estudio. Los siguientes hallazgos fueron informados: mucosa normal, correspondiente a la presencia de mucosa fúndica con células parietales, sin evidencias de proceso inflamatorio agudo o crónico. Funditis: se definió como la presencia de mucosa fúndica con elementos celulares de tipo inflamatorio ya sea poli o mononucleares. Carditis: corresponde a la presencia de mucosa cardial, con células columnares y asociación de un proceso inflamatorio crónico.

También se determinó la presencia de Helicobacter pylori en estas muestras yuxtacardiales.

Técnica quirúrgica. El tipo de cirugía bariátrica que se empleó corresponde a un bypass gástrico reseccional ${ }^{18,19}$, que consiste en crear una bolsa gástrica proximal, con una capacidad total menor a $20 \mathrm{ml}$ y un asa en Y-de-Roux de 125 a $150 \mathrm{~cm}$ por vía abierta.

Seguimiento. Todos los pacientes fueron controlados clínicamente por los autores a los 6, 12 y 24 meses de la operación, evaluando la presencia de síntomas de reflujo gastroesofágico, determinando la baja de peso, los cambios en la calidad de vida y en la comorbilidad. Estos dos últimos aspectos están evaluados en otra publicación ${ }^{19}$.

Estadísticas. Se empleó el test exacto de Fisher y el Chi cuadrado, tomando un $\mathrm{p}<0,05$ como significativo.

\section{RESUlTAdos}

En el presente estudio, la evolución postoperatoria fue muy satisfactoria en 66 de los 69 pacientes (96\%) y sólo 3 presentaron una complicación postoperatoria menor. El tiempo promedio de seguimiento fue de 21 meses (rango 12 a 50 meses). En la Tabla 1 se muestra la presencia de síntomas de reflujo GE antes y 21 meses después de la cirugía. Se aprecia que todos los pacientes presentaban pirosis, regurgitación o ambos. Después de la cirugía, 93\% estaba asintomático a 2 años del control. El IMC presentó también una disminución significativa de 40\%, lo que corresponde a $72 \%$ de pérdida de exceso de peso $(\mathrm{p}<0,001)$. En la Tabla 2 se señalan los hallazgos endoscópicos antes y después de la cirugía. Esofagitis erosiva 
Tabla 1. H allazgos clínicos en 69 pacientes sometidos a bypass gástrico 21 meses después de la cirugía

\begin{tabular}{|lllll|}
\hline & & Preoperatorio & Postoperatorio & $\mathrm{p}$ \\
\hline I. & Síntomas & & & \\
& Pirosis & $65(94 \%)$ & $3(4,3 \%)$ & $<0,000$ \\
& Regurgitación & $49(71,0 \%)$ & $1(1,4 \%)$ & $<0,000$ \\
II. & Indice de masa & & & $<0,001$ \\
& corporal $\left(\mathrm{kg} / \mathrm{m}^{2}\right)$ & $47,7 \pm 5,4$ & $28,7 \pm 4,1$ & \\
& & Rango 35-63 & Rango 22-44 & \\
\hline
\end{tabular}

Tabla 2. H allazgos endoscópicos de esofagitis en 69 pacientes con obesidad mórbida sometidos a bypass gástrico antes y 21 meses después de la cirugía

\begin{tabular}{|c|c|c|c|c|}
\hline & & Preoperatorio & Postoperatorio & $\mathrm{p}$ \\
\hline \multirow[t]{3}{*}{ I. } & Grupo total & & & \\
\hline & Esofagitis erosiva & $67(97 \%)$ & $5(7,2 \%)$ & $<0,001$ \\
\hline & Ulcera esofágica & $2(3 \%)$ & 0 & \\
\hline \multirow[t]{6}{*}{ II. } & Según IMC $\left(\mathrm{kg} / \mathrm{m}^{2}\right)$ & & & \\
\hline & $35-39,9(n=15)$ & Esofagitis $15(100 \%)$ & $2(13,3 \%)$ & $<0,001$ \\
\hline & $40-49,9(n=46)$ & Esofagitis 45 (98\%) & $2(4,3 \%)$ & $<0,001$ \\
\hline & & Ulcera 1 & - & \\
\hline & $\geq 50 \quad(n=8)$ & Esofagitis 7 (88\%) & $1(12,5 \%)$ & $<0,001$ \\
\hline & & Ulcera 1 (12\%) & - & \\
\hline
\end{tabular}

estaba presente en 97\% y úlcera esofágica en 2 pacientes (3\%). En el control alejado, 5 pacientes $(7,2 \%)$ presentaban erosiones esofágicas, siendo 2 de ellos asintomáticos. En los 2 pacientes que tenían úlcera esofágica, ésta sanó. No hubo ninguna diferencia en la respuesta esofágica al bypass gástrico según el IMC preoperatorio. En la Tabla 3 se muestran los hallazgos histológicos antes y después de la cirugía a nivel de las biopsias yuxtacardiales. No hubo diferencia significativa en ninguno de los hallazgos histológicos. Las biopsias catalogadas como normales correspondieron a mucosa fúndica o cardial sin inflamación. $\mathrm{H}$ pylori estaba presente en 16\% de los pacientes antes de la cirugía y 34\% después de la cirugía.

\section{DisCUSIÓN}

El presente estudio clínico y endoscópico demuestra que el bypass gástrico es efectivamente la
Tabla 3. H allazgos histológicos a nivel yuxtacardial en pacientes con obesidad mórbida sometidos a bypass gástrico antes y 21 meses después de la cirugía

\begin{tabular}{|lccc|}
\hline & $\begin{array}{c}\text { Preoperatorio } \\
\mathrm{n}=69\end{array}$ & $\begin{array}{c}\text { Postoperatorio } \\
\mathrm{n}=23\end{array}$ & $\mathrm{p}$ \\
\hline Normal & $45(65,2 \%)$ & $13(56 \%)$ & $\mathrm{ns}$ \\
Funditis & $10(14,5 \%)$ & $8(35 \%)$ & $\mathrm{ns}$ \\
Carditis & $14(20,3 \%)$ & $2(9 \%)$ & $\mathrm{ns}$ \\
H pylori & $11(16 \%)$ & $8(34 \%)$ & $\mathrm{ns}$ \\
\hline
\end{tabular}

ns $=$ no significativo.

mejor operación antirreflujo conocida, con resultados muy superiores a una fundoplicatura clásica de Nissen o la calibración cardial con gastropexia posterior.

La primera observación de este efecto se remonta a $1982^{20}$, cuando Buckwalter observó 
que 6 pacientes con obesidad mórbida sometidos a bypass gástrico, con agregado de vagotomía troncal y piloroplastia, mejoraron todos en forma significativa, evaluando síntomas de reflujo GE. Posteriormente, Jones en $1991^{12}$ estudió a 100 pacientes sometidos a bypass gástrico, notando que $98 \%$ presentaban ausencia de síntomas 1 año después de la cirugía, mientras que 2 presentaban síntomas leves. Desde entonces, este particular aspecto ha sido de gran interés para numerosos autores. El efecto de la cirugía del bypass gástrico sobre la presencia de esofagitis y reflujo GE crónico patológico se ha evaluado de 3 maneras: mediante el análisis de síntomas, por hallazgos endoscópicos y por los resultados del pH de $24 \mathrm{~h}$.

El análisis clínico ha sido lo más comunicado dada la facilidad de su análisis, ya que no requiere de ningún examen, salvo el interrogatorio clínico. Tiene la desventaja que es un análisis subjetivo, que no siempre se correlaciona con la real presencia de reflujo gastroesofágico ${ }^{21}$. En la literatura internacional hay 8 estudios que se refieren exclusivamente a evaluación clínica de síntomas antes y después del bypass gástrico. Smith y cols ${ }^{10}$ estudiaron a 188 pacientes sometidos a bypass gástrico, teniendo 34 de ellos síntomas de reflujo gastroesofágico. Después de la cirugía sólo un paciente $(3 \%)$ persistía con pirosis. Jones y cols en 2 estudios ${ }^{11,12}$ evaluaron a 44 y 100 pacientes, respectivamente, demostrando que $98 \%$ de los pacientes se hicieron asintomáticos después de la cirugía. Kim y $\operatorname{cols}^{13}$ relatan 2 pacientes con banda gástrica vertical y esofagitis severa que mejoraron con el bypass gástrico. Clements y cols ${ }^{14}$ evaluaron a 36 pacientes, teniendo $32 \%$ de ellos pirosis, que persistió en 11,8\% después de la cirugía. Fister y cols ${ }^{15}$ evaluaron 35 pacientes a 6 meses de la cirugía, notando en todos desaparición de síntomas de RGE.

Frezza y cols ${ }^{17}$ analizaron 66 pacientes sometidos a bypass gástrico laparoscópico, notando una disminución de la pirosis de $87 \%$ a $22 \%$ y de la regurgitación de $45 \%$ a $22 \%$. Ortega y cols ${ }^{22}$ en 40 pacientes describen presencia de pirosis, $60 \%$, que mejoró significativamente con el bypass gástrico, quedando $12,5 \%$ con síntomas. Nos llama la atención la alta prevalencia de pirosis postoperatoria en algunos estudios como los de Clements, Frezza y Ortega, mientras que otros comunicados, similares al nuestro, demostraron persistencia de pirosis en sólo 3\% de los pacientes. Hay un estudio que demostró que la fundoplicatura de Nissen ${ }^{16}$ tiene $31 \%$ de recurrencia en obesos con reflujo GE y $54 \%$ de recurrencia si se realiza por vía intratorácica.

La evaluación de reflujo ácido patológico por pHmetría de $24 \mathrm{~h}$ ha sido medida antes de la cirugía en 3 estudios ${ }^{3,22,23}$, demostrando alta prevalencia de reflujo ácido en pacientes con obesidad mórbida. Sin embargo, después de la cirugía sólo hay un estudio que se refiere a este aspecto $^{22}$. En este trabajo, el tiempo total, en que el esofágo permanece a un $\mathrm{pH}<4$ en $24 \mathrm{~h}$, fue de $4,9 \%$ antes de la cirugía y de $2,4 \%$ después del bypass gástrico. Hubo $57 \%$ de los pacientes evaluados con pHmetría alterada antes de la operación, persistiendo sólo $15 \%$ con reflujo ácido positivo.

Estudios endoscópicos, como el presente, son muy escasos en la literatura internacional referente a bypass gástrico. Balsinger y $\operatorname{cols}^{24}$ analizaron a 25 pacientes con banda gástrica vertical fallida que fueron convertidos a bypass gástrico. Catorce pacientes tenían esofagitis erosiva antes de la cirugía (56\%). Se pudo realizar endoscopia en sólo 5 pacientes después de la cirugía (36\%), demostrando desaparición de la esofagitis en todos. El presente estudio demuestra que la evaluación endoscópica del paciente con esofagitis erosiva al control endoscópico alejado, muestra una mejoría significativa con desaparición de la esofagitis en $95 \%$ de los pacientes. La úlcera esofágica en 2 pacientes cicatrizó completamente. De los 4 pacientes con esofagitis erosiva sólo 2 presentaron síntomas. Los 4 pacientes están en la actualidad con terapia médica continua con inhibidores de la bomba de protones. La presencia de $\mathrm{H}$ pylori pareció duplicarse después de la operación a nivel yuxtacardial, pero sin significación estadística. No tenemos una explicación clara para este hallazgo, que pudiera corresponder sólo a un número diferente de pacientes evaluados en el postoperatorio comparado al análisis de la intervención.

La pregunta importante que surge se refiere al mecanismo de por qué el bypass gástrico, independiente de la resección o no del segmento gástrico distal, es la mejor técnica antirreflujo actualmente conocida. La respuesta es prácticamente la misma que hemos dado para el efecto de la operación de supresión ácida y derivación 
duodenal en pacientes con esófago de Barrett extenso $^{25,26}$. Con esta operación, la vagotomía y gastrectomía parcial disminuye la secreción ácida en $90 \%$ y el asa en Y de Roux elimina completamente el esófago de contenido duodenal. Al abolir el material refluido que daña la mucosa esofágica (secreción ácida gástrica y contenido duodenal), los pacientes con esófago de Barrett extenso mejoraron significativamente con el bypass gástrico. Con este tipo de cirugía bariátrica, por la enorme reducción de la masa de células

\section{REFERENCIAS}

1. Barak N, Ehrenpreis ED, Harrison JR, Sitrin MD. Gastroesophageal reflux disease in obesity: pathophysiological and therapeutic considerations. Obes Rev 2002; 3: 9-15.

2. Gómez O, Herrera mF, Valdovinos ma. Obesidad y enfermedad por reflujo gastroesofágico. Rev Invest Clin 2002; 54: 320-7.

3. Csendes A, Burdiles P, Rojas J, Burgos A, Henríquez A. Reflujo gastroesofágico patológico en pacientes con obesidad severa, mórbida e hiperobesidad. Rev Méd Chile 2001; 129: 1038-41.

4. Korn O, Puente G, Sagastume H, Burdiles P, Csendes A. Reflujo gastroesofágico y obesidad. Rev Méd Chile 1997; 125: 671-5.

4. Clements RM, González QM, Foster A, Richards WO, McDowell J, Bandora A et al. Gastrointestinal symptoms are more intense in morbidly obese patients and are improved with laparoscopic Roux-en-Y gastric bypass. Obes Surg 2003; 13: 610-4.

5. Brouln RE. Gastric bypass. Surg Clin N Am 2001; 81: 1077-91.

6. Cottam DR, Mattan SG, Schauer PR. Laparoscopic era of operations for morbid obesity. Arch Surg 2003; 138: 367-75.

7. Weber M, Muller MiC, Bucher T, Weldi S, Drido D, HoRber F ET AL. Laparoscopic gastric bypass is superior to laparoscopic gastric banding for treatment of morbid obesity. Ann Surg 2004; 240: 97583.

8. Deite M. Overview of operations for morbid obesity. World J Surg 1998; 22: 913-8. parietales, quedando una capacidad gástrica total de menos de $20 \mathrm{ml}$, la secreción ácida disminuye en forma muy significativa. Hay dos estudios que han medido este parámetro ${ }^{17,18}$, demostrando una reducción de la secreción basal de 5 a $0,01 \mathrm{mEq} / \mathrm{h}$ de la secreción estimulada de 12,1 a 0,08 mEq/h. Además el asa larga en Y de Roux de 125 a 150 cm elimina completamente el reflujo de contenido intestinal. De esta manera, el bypass gástrico resulta ser la mejor operación "antirreflujo" que existe en la actualidad.

9. Fobi MAL, Lee H, Holness R, Cabrinda DG. Gastric bypass operation for obesity. World J Surg 1998; 22: 925-35.

9. Patterson EJ, Davis DG, Khajanchee Y, Swanstrom LL. Comparison of objective outcomes following laparoscopic Nissen fundoplication vs laparoscopic gastric bypass in the morbidly obese with heartburn. Surg Endosc 2003; 17: 1565.

10. Smith SHC, Edwards CB, Goodman GN. Symptomatic and clinical improvement in morbidly obese patients with gastroesophageal reflux disease following Roux en Y gastric bypass. Obes Surg 1997; 7: 479-84.

11. Jones KB. Roux en Y gastric bypass: an effective antireflux procedure in the less than morbidly obese. Obes Surg 1998; 8: 35-8.

12. Jones KB, Allen TV, Manas KS. Roux en Y gastric bypass: an effective antireflux procedure. Obes Surg 1991; 1: 295-8.

13. Kim CHH, SARR HG. Severe reflux esophagitis after vertical banded gastroplasty for treatment of morbid obesity. Mayo Clinic Prob 1992; 67: 33-5.

14. Clements RM, González QH, Foster A, Richards WO, Mc Dowells J, Bardora A, Laws HL. Gastrointestinal symptoms are more internal in morbidly obese patients and are improved with laparoscopic Roux en Y gastric bypass. Obes Surg 2003; 13: 610-4.

15. Foster A, Laws HL, González QH, Clements RH. Gastrointestinal symptomatic outcome after laparoscopic Roux-en-Y gastric bypass. J Gastroent Surg 2003; 7: 750-3.

16. Pérez AR, Moname AC. Rattner DW. Obesity adversely effects the outcome of antireflux operations. Surg Endosc 2001; 15: 986-9. 
17. Frezza eE, Inramuddin S, Gourash W, Rakite T, Kingston A, Luketich J, Schauer PR. Symptomatic improvement in gastroesophageal reflux disease following laparoscopic Roux en Y gastric bypass. Surg Endosc 2002; 16: 1027-31.

18. Csendes A, Burdiles P, Díaz JC, Maluenda F, Burgos AM, Recio M et al. Resultados del tratamiento quirúrgico de la obesidad mórbida. Análisis de 180 pacientes. Rev Chil Cir 2002; 54: 3-9.

19. Csendes A, Burdiles P, Papapietro K, Díaz JC, Maluenda F, Burgos A et al. Results of gastric bypass plus resection of the distal excluded gastric segment in patients with morbid obesity. J Gastroent Surg 2005; 9: 121-31.

20. Buckwalter JA. Surgical treatment of morbid obesity with reflux esophagitis. Amer Surg 1982; 48: 128-30.

21. Csendes A, Rencoret G, Beltrán M, Smok G, Henríquez A. Correlación entre síntomas de reflujo gastroesofágico y resultado de la pHmetría de $24 \mathrm{~h}$ en pacientes con estudio endoscópico normal o levemente alterado. Rev Méd Chile 2004; 132: 19-25.

22. Ortega J, Escudero MD, Mora F, Sala C, Flor B, Martínez J ET AL. Outcome of esophageal function and 24 hours esophageal $\mathrm{pH}$ monitoring after vertical banded gastroplasty and Roux-en-Y gastric bypass. Obes Surg 2004; 14: 1086-94.
23. Suter M, Dorta G, Giusti V, Calmes JM. Gastroesophageal reflux and esophageal motility disorders in morbidly obese patients. Obese Surg 2004; 14: 956-66.

24. Balsiger BM, Murr MM, Mai J, Sarr MG. Gastroesophageal reflux after intact vertical banded gastroplasty. Correction by conversion to Roux en Y gastric bypass. J Gastroent Surg 2000; 4: 27681.

25. Csendes A, Burdiles P, Braghetto i, Korn O, Díaz JC, Rojas J. Early and late results of the acid suppression and duodenal diversion operation in patients with Barrett's esophagus. World J Surg 2004; 26: 566-76.

26. Csendes A, Braghetto I, Burdiles P, Korn O. Roux en $\mathrm{Y}$ lumb diversion as the first option for patients who have Barrett's esophagus. Chest Surg Clin N Am 2002; 12: 157-84.

27. Smith CD, Herkes SHB, Behrns KE, Fairbanks VF, Kelly KA, SARr MG. Gastric acid secretion and vitamin B12 absorption after vertical Roux en Y gastric bypass for morbid obesity. Ann Surg 1993; 218: 91-6.

28. Benrs KE, Smith CD, SARr MG. Prospective evaluation of gastric acid secretion and cobalmin absorption following gastric bypass for clinically severe obesity. Dig Dis Sci 1994; 39: 315-20. 